\title{
Spontaneous High Piezoelectricity in Poly(vinylidene fluoride) Nanoribbons Produced by Iterative Thermal Size Reduction Technique
}

\author{
Mehmet Kanik, ${ }^{t, \neq}$ Ozan Aktas, ${ }^{+, \S}$ Huseyin Sener Sen, ${ }^{\dagger}$ Engin Durgun, ${ }^{t, \neq}$ and Mehmet Bayindir ${ }^{t, \neq, s, *}$ \\ ${ }^{\dagger}$ UNAM-National Nanotechnology Research Center, Bilkent University, 06800 Ankara, Turkey, ${ }^{\ddagger}$ Institute of Materials Science and Nanotechnology, Bilkent University, \\ 06800 Ankara, Turkey, and ${ }^{\S}$ Department of Physics, Bilkent University, 06800 Ankara, Turkey
}

\begin{abstract}
We produced kilometer-long, endlessly parallel, spontaneously piezoelectric and thermally stable poly(vinylidene fluoride) (PVDF) micro- and nanoribbons using iterative size reduction technique based on thermal fiber drawing. Because of high stress and temperature used in thermal drawing process, we obtained spontaneously polar $\gamma$ phase PVDF micro- and nanoribbons without electrical poling process. On the basis of X-ray diffraction (XRD) analysis, we observed that PVDF micro- and nanoribbons are thermally stable and conserve the polar $\gamma$ phase even after being exposed to heat treatment above the melting point of PVDF. Phase transition mechanism is investigated and explained using
\end{abstract}

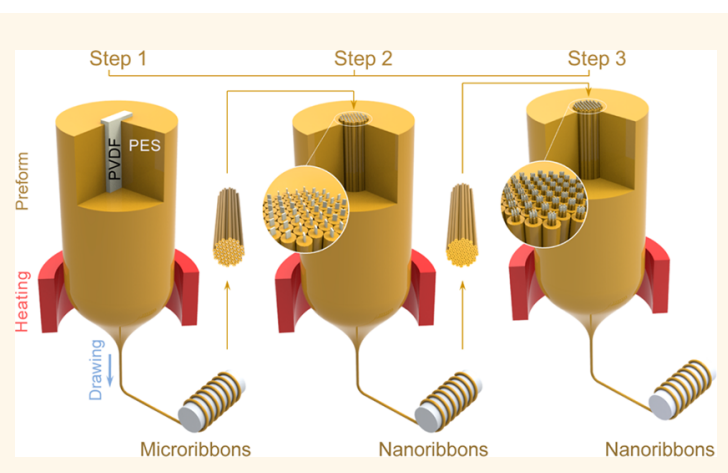
ab initio calculations. We measured an average effective piezoelectric constant as $-58.5 \mathrm{pm} / \mathrm{V}$ from a single PVDF nanoribbon using a piezo evaluation system along with an atomic force microscope. PVDF nanoribbons are promising structures for constructing devices such as highly efficient energy generators, large area pressure sensors, artificial muscle and skin, due to the unique geometry and extended lengths, high polar phase content, high thermal stability and high piezoelectric coefficient. We demonstrated two proof of principle devices for energy harvesting and sensing applications with a $60 \mathrm{~V}$ open circuit peak voltage and $10 \mu \mathrm{A}$ peak short-circuit current output.

KEYWORDS: piezoelectric polymer · nanoribbon · fiber drawing · PVDF · energy harvesting · $a b$ initio calculation

U tilization of the unique material properties represented by flexible, lightweight and biocompatible piezoelectric polymeric materials such as poly(vinylidene fluoride) $(P V D F)^{1}$ and its copolymer poly(vinylidene fluoride trifluoroethylene) (PVDF-TrFE) ${ }^{2}$ is expected to bring on new horizons for sensor, actuator, and energy harvesting applications where piezoelectric ceramic materials have been employed hitherto. A closer look into the applications of piezoelectricity including biosensing, ${ }^{3}$ energy generation, ${ }^{4-6}$ pressure sensing, ${ }^{7}$ high precision positioning, ${ }^{8}$ artificial muscle and $\mathrm{skin}^{9,10}$ reveals that thermally stable, flexible, and stretchable piezoelectric materials are required to be produced with high yields and in a cost-effective way for the fabrication of commercially feasible, large area, self-powering, and highly efficient devices. Although ceramic piezoelectric materials can present higher piezoelectric coefficients, they suffer from high brittleness, low cyclic endurance, high processing temperature and high production cost as well as toxic elemental composition in contrast to properties of polymer piezoelectric materials. ${ }^{11,12}$

Since the discovery of PVDF $\left(\mathrm{CH}_{2}-\mathrm{CF}_{2}\right)_{n \text {, }}$ which has the highest piezoelectric coefficient among all polymers, ${ }^{1}$ PVDF and copolymers have been a subject of intense research, which initiated development of many fabrication methods for PVDF thin film ${ }^{13}$ and nanowires. ${ }^{14}$ Regardless of the production method, piezoelectric properties are dependent on the amount of polar phase of PVDF. ${ }^{15,16}$ Four commonly known forms of PVDF are $\alpha, \beta, \gamma$ and $\delta$ phases, which are represented by different stereochemical structures with alternating s-trans and s-gauge $\mathrm{C}-\mathrm{C}$ bonds (TGTG, $\Pi \Pi T$, $\Pi \pi G T T \bar{G}$, and $\left.T G T \bar{G}_{(\text {polar })}\right)$, respectively. ${ }^{15,17}$
* Address correspondence to bayindir@nano.org.tr.

Received for review June 16, 2014 and accepted August 18, 2014.

Published online August 18, 2014 $10.1021 / \mathrm{nn} 503269 \mathrm{~b}$

() 2014 American Chemical Society 
The most stable and easily obtained form of PVDF is the nonpolar $\alpha$ phase, which can also be in a rarely found polar form named $\delta$ phase. On the other hand, $\beta$ and $\gamma$ phases are the most desirable forms of PVDF due to their high polar molecular conformations. ${ }^{14,18-21} \mathrm{High}$ polarity of the $\beta$ phase as a result of all trans $\mathrm{C}-\mathrm{C}$ bonds makes PVDF a promising candidate for piezoelectric, pyroelectric and ferroelectric applications., $14,18,19$ Transitions between phases of PVDF are possible under specific nucleation and processing conditions (Figure S1, Supporting Information (SI)). Irrespective of the initial phase, the amount of the $\beta$ phase in PVDF thin films can be increased by adding inorganic additives or stretching more than $80 \%$ at $95{ }^{\circ} \mathrm{C}$ following with an electrical poling. ${ }^{15,22}$ Transition mechanisms resulting in $\gamma$ phase are crystallization at high temperature or applying high shear on molten polymer, long-term annealing at high temperature or polling at low electric field from $\alpha$ phase, and low rate evaporation from high temperature polymer solutions. ${ }^{16,20,21,23}$ Although, $\gamma$ phase has lower polarity when compared to $\beta$ phase, its piezoelectric coefficient still stands better than those of other polymers. ${ }^{17,20}$ Besides, the higher Curie temperature of $\gamma$ phase makes it less vulnerable to harsh environmental conditions. Because of the decay of remnant polarization of $\beta$ phase PVDF and PVDF-TrFE at lower Curie temperatures, these materials are not good candidates for production of thermally stable long endurance devices. ${ }^{24}$ Because of its superior properties, $\gamma$ phase is highly desirable. Unfortunately, it is known to be hard to access experimentally. ${ }^{20}$

The most common way of triggering polar phase transitions is applying a high strength electric field at elevated temperatures. ${ }^{14,25,26}$ In addition, diminishing the feature size of PVDF lowers the cost and energy required for the polarization process. ${ }^{27}$ In accordance with the developments in nanotechnology, scientists have been trying to facilitate nanoscale effects to improve the piezoelectric properties of PVDF and copolymers by altering their composition, ${ }^{21,22,28,29}$ size and shape. ${ }^{18-20,23,27,30,31}$ Initial attempts were focused on developing PVDF piezoelectric thin films by using spray coating, ${ }^{32}$ spin coating, ${ }^{17}$ physical vapor deposition, $^{33}$ and the Langmuir-Blodgett thin film coating methods. ${ }^{34}$ Although shaping the PVDF in the form of thin film can disclose elevated piezoelectric properties, PVDF nanowires lead to superior piezoelectricity, due to the one-dimensional nanoscale confinement. ${ }^{7,14}$ Results of nanoconfinement effects such as spontaneous polarization can eliminate external electrical poling process for polar phase transition. ${ }^{19,20,31}$

Beyond the controversy, nanowires have made great impacts on many disciplines including solar cells, biosensors and phase change memory devices. ${ }^{35-38}$ A similar impact is also expected on piezoelectric applications using PVDF nanowires. The prominent piezoelectric PVDF nanowire fabrication methods are anodized alumina (AAO) template molding, ${ }^{19,20,39}$ electrospinning, 7,14,40,41 and nanoimprint lithography $(\mathrm{NI}),{ }^{18,23,27,31}$ which are all solvent dependent. Even though these techniques are appropriate to produce PVDF nanowires, they are not superior in all aspects considering the nanowire aspect ratio, uniformity, geometry control, yield, and device integrability in order to produce large area, low cost and high throughput devices (Table S1 (SI)). Despite the fact that high aspect ratio nanowires can be produced with high yield by using electrospinning, diameter uniformity and geometry control capability of this technique are not fulfilling the requirements of current state of the art technology. Tuning the diameter of nanowires can be better accomplished by using AAO and $\mathrm{NI}$ techniques. However, nanowires produced by these methods are not feasible to carry out the production of flexible, large area devices. Above all, unlike electrospinning, AAO, and NI techniques, a fabrication technique that provides PVDF nanowire production with high aspect ratio, excellent uniformity, desired geometry, and high yield in a multimaterial fashion can pave the way for in situ assembly of nanowires with metallic, semiconductor and dielectric components of the macroscopic devices. ${ }^{42,43}$

\section{RESULTS AND DISCUSSION}

Compatibility of iterative fiber drawing based size reduction technique for a wide range of materials in nanowire shape has been demonstrated. ${ }^{43-45}$ On the contrary to a recent study, ${ }^{46}$ it is possible to produce micro- and nanostructured PVDF in polar phase spontaneously induced by fiber drawing process as we showed in this study for the first time. Here, we produced kilometer-long, PVDF arrays in novel forms such as nanoribbon and square cross-sectional nanoshell (Figure S2 (SI)) structures. We rather prefer ribbon shape with planar contact areas, which poses a unique advantage for piezoelectric measurements, unlike the nanowires with cylindrical symmetry. We achieved highly polar $\gamma$ phase PVDF nanoribbons from the fiber drawing technique simultaneously utilizing high temperature and shear stress triggered by tensile forces (Figure S3 and Video S1 (SI)). In order to simulate the phase transformation in iterative fiber drawing process, we performed $a b$ initio calculations based on density functional theory in which the effects of both temperature and shear stress are included. The details of the method are explained in the Supporting Information. We compared the phase transition at 0 and $470 \mathrm{~K}$ (above the melting temperature of PVDF) from $\alpha$ and $\beta$ phase to $\gamma$ phase under varying compressive and tensile strain. $\gamma$ phase in the as produced PVDF nanoribbons is observed to still exist after annealing at high temperatures up to $175^{\circ} \mathrm{C}$. $\alpha, \beta$ and $\gamma$ phase distribution in PVDF ribbons are investigated as a function of size using X-ray diffraction (XRD) and attenuated total 
reflectance-fourier transform infrared (ATR-FTIR) spectroscopy techniques. PVDF ribbons are confirmed by morphological characterizations conducted with scanning electron microscopy (SEM). Using a piezo evaluation system attached to an atomic force microscope (AFM), we observed a large piezoelectric response $\left(d_{33}=-58.5 \mathrm{pm} / \mathrm{V}\right)$ from a single $80 \mathrm{~nm}$ thick nanoribbon. To our knowledge, this is the first piezoelectric measurement performed on a single PVDF nanoribbon. Besides, here we report the highest effective $d_{33}$ coefficient ever measured from a $\gamma$ phase single PVDF nanoribbon. To utilize the superior performance, we designed two different device structures for the purpose of energy harvesting and tapping sensor. Peak voltage and current outputs of our devices are measured as $60 \mathrm{~V}$ and $10 \mu \mathrm{A}$.

The nanoribbon fabrication procedure starts with preparation of a multimaterial preform, which is an exact macroscopic copy of the final fibers. The easiest way to produce a preform is rolling polymer films, degassing the air trapped in the roll under a vacuum at a temperature below the glass transition temperature of the films and last consolidating in a vacuum oven at the glass transition temperature of the films. Following the mentioned procedure, a PVDF preform is prepared by rolling $60 \mu \mathrm{m}$ thick PVDF films around a glass tube and consolidated in a tube oven at $180^{\circ} \mathrm{C}$ for $30 \mathrm{~min}$ under a $2 \times 10^{-2}$ Torr vacuum. Next, a slab $(3 \mathrm{~mm} \times$ $10 \mathrm{~mm} \times 10 \mathrm{~cm}$ ) is mechanically extracted from this PVDF preform. Afterward, a poly(ether sulfone) (PES) preform with $35 \mathrm{~mm}$ in diameter and $25 \mathrm{~cm}$ in length is made by rolling $100 \mu \mathrm{m}$ thick PES films around a $3 \mathrm{~mm}$ glass tube and consolidated at $255^{\circ} \mathrm{C}$ for $35 \mathrm{~min}$ under a $2 \times 10^{-2}$ Torr vacuum. The PES preform is split in two halves and machined in the center to open a niche for inserting the PVDF slab (Figure 1a).

Fiber drawing processes are executed in a custommade fiber tower, which consists of a vertical tube oven, a preform feeding mechanism, and a real time monitoring system measuring applied stress, temperature, and thickness of the drawn fiber (Figure S4 (SI)). Figure $1 \mathrm{~b}$ summarizes the nanoribbon fabrication technique. The fabrication of PVDF nanoribbon arrays, which comprises several fiber drawing steps, starts with the drawing of PVDF slab embedded PES preform. Heating the preform above the glass transition temperature of PES and melting point of PVDF and applying approximately $3 \mathrm{MPa}$ tensile stress are required in order to trigger plastic deformation in fiber drawing process. Since drawing temperature $\left(285^{\circ} \mathrm{C}\right)$ or the temperature in the core of the preform $\left(\sim 200^{\circ} \mathrm{C}\right)$ is higher than the melting point of PVDF $\left(T_{m}=165^{\circ} \mathrm{C}\right)$, the molten slab flows and shrinks in the PES cladding during fiber drawing. The first step of thermal drawing process results in PVDF microribbons with various thicknesses ranging from 400 to $10 \mu \mathrm{m}$ by adjusting the process temperature, applied stress, drawing and feeding speed. Figure 2a shows as drawn step 1 fibers and Figure $2 \mathrm{~b}$ represents cross sectional and longitudinal SEM images of $30 \mu \mathrm{m}$ thick microribbon embedded fibers, which are produced in the first step with a preform feeding speed $8 \mathrm{~mm} / \mathrm{sec}$. For the second drawing step, approximately 400 first step fibers are stacked and inserted into cylindrical hollow core of a new PES preform, which becomes a multifunctional jacket protecting and keeping the PVDF nanoribbons together. Sequential thermal drawings of the preform prepared for the second step result in thicknesses ranging from 300 to $50 \mathrm{~nm}$ (Figure 2c,d). Similarly, 400 nanoribbon embedded fibers obtained in previous steps are inserted in new PES preforms produced for the third step fiber drawing processes. After drawing the third step preform, nanoribbons result in thicknesses ranging from 50 to $5 \mathrm{~nm}$ (Figure 2e,f). We extracted PVDF nanoribbons out of the PES cladding by using dichloromethane (DCM), which cannot dissolve PVDF. Cross sectional characterization of nanoribbons requires a special sample preparation using ultramicrotome for SEM imaging. However, diamond knife of the ultramicrotome causes defects that hamper observing the exact cross sectional shape of the nanoribbons. In addition, even though state of the art electron microscopy techniques are used, imaging of nanometer scale polymer features is very difficult due to fast degradation of polymers under high energy electron beam. ${ }^{47}$ Alternatively, a better SEM observation of the ribbon shape is accomplished using two different sample preparation methods: breaking the fibers after a liquid nitrogen treatment (Figure S5 (SI)) and direct cutting of the fibers longitudinally (Video S2 (SI)). Size distribution for second step PVDF nanoribbons is given in Figure S6 (SI). Standard deviation normalized with respect to the mean of the nanoribbons size distribution is found to be $\sim 11 \%$.

The distance between nanoribbons is well-defined with cladding thicknesses of the input fibers. In addition, the macroscopic encapsulation polymer makes it practicable to manually manipulate kilometers long, perfectly aligned, millions of nanowires. Number and total lengths of the nanoribbon embedded fibers are progressive in conformity with the growing number of drawing steps. For instance, despite that a $200 \mathrm{~m}$ long in-fiber single ribbon is obtained after the first step drawing, nanoribbons are achieved in kilometers of length and thousands of number in following drawing steps. Dimensions of the first step slab and preform, overall reduction ratio, and the number of steps in iterative fiber drawing processes determine the final size of nanoribbons. Besides, ribbon shape is inherited by next generation ribbons from the fibers produced in previous steps.

Molecular conformation of $\alpha, \beta$ and $\gamma$ phases are shown in Figure 3a. Predominance of the polar $\gamma$ phase 

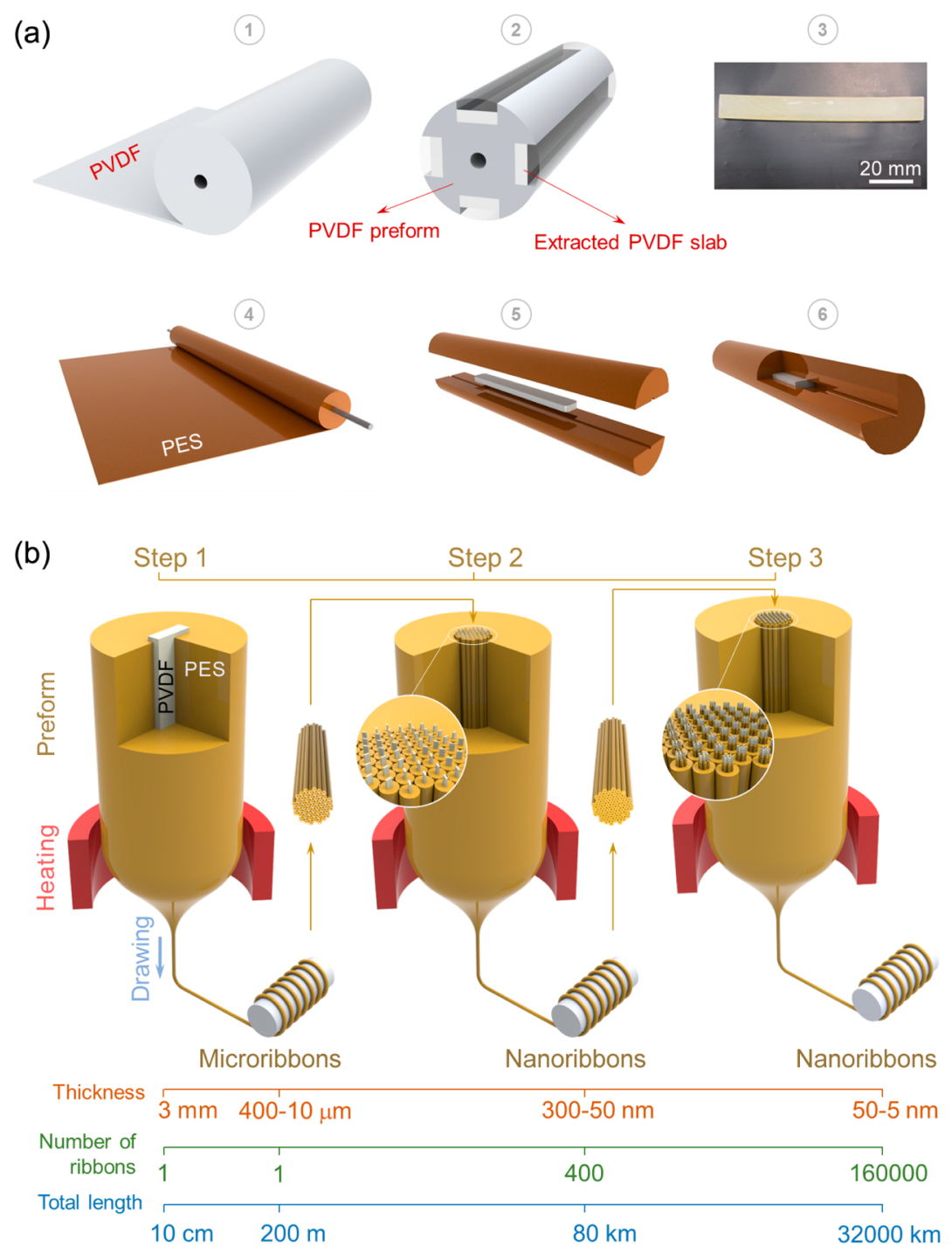

Figure 1. A novel top-to-bottom nanofabrication technique for producing kilometer-long piezoelectric nanoribbons. (a) The fabrication steps of the PVDF ribbon embedded in PES preform, which is a macroscopic copy of the final nanoribbons. As an initial step, (1) a PVDF preform is built by rolling PVDF sheets, and (2-3) a slab is mechanically extracted out of the PVDF preform. (4-5) Next, the PVDF slab is inserted into a PES preform, which is then sealed by heating in a consolidator oven. (6) The final multimaterial product turns into a PVDF ribbon embedded PES matrix. Dimensions and design specifications of the preform directly affect the size and shape of the final product of the drawing process. (b) An iterative fiber drawing scheme allows to achieve nanometer structures. A macroscopic PVDF slab is inserted, thermally sealed in a PES matrix, and drawn thermally to produce first step fibers. At high temperatures, molten PVDF flows together with softened PES cladding, producing tens of meters long PVDF microribbon encapsulated in PES. First step fibers are stacked and redrawn in a new preform for the second step fiber drawing in order to decrease the size of the ribbons down to nanometers. Further size reduction can be accomplished by following same stacking and redrawing cycles. From the first step to the last step, total number and length of the ribbons in the fiber increase as the size of each nanoribbon decreases.

ribbons produced in first, second, and third drawing steps is clearly observed by using XRD. PES claddings of nanoribbons are etched in DCM before XRD measurements in order to eliminate amorphous background arising from the PES cladding, and to increase the signal intensity. In the literature, characteristic peak positions (2 $\theta$ ) of PVDF are tabulated as $17.7^{\circ}, 18.4^{\circ}$, $19.9^{\circ}, 26.5,27.8^{\circ}, 35.7^{\circ}, 39^{\circ}$, and $57.4^{\circ}$ for $\alpha$ phase; $20.7^{\circ}, 20.8^{\circ}, 35^{\circ}, 36.6^{\circ}$, and $56.1^{\circ}$ for $\beta$ phase; $18.5^{\circ}$, $19.2^{\circ}, 20.1^{\circ}, 20.3^{\circ}, 26.8^{\circ}, 36.2^{\circ}$, and $38.7^{\circ}$ for $\gamma$ phase. ${ }^{15,16,20,21}$ Starting from the PVDF macroscopic slab, we have analyzed change of phase content for all fabrication steps (Figure $3 b)$. The slab includes a minor amount of $\beta\left(2 \theta=20.7^{\circ}\right)$ and $\alpha\left(2 \theta=27.8^{\circ}\right)$ phases, and a transition trend to the $\gamma$ phase is persistent starting from the first step ribbons. Although suppressed $\alpha$ phase peaks still exsit at $17.7^{\circ}, 26.5^{\circ}$ and $27.8^{\circ}$ peak positions, dominance of the $\gamma$ phase is obvious from the peaks at $2 \theta=18.5^{\circ}, 20.1^{\circ}$ and $26.8^{\circ}$. For the second step nanoribbons, except shifts observed in $\gamma$ peak positions from $20.1^{\circ}$ to $20.3^{\circ}$, and $18.5^{\circ}$ to $18.6^{\circ}$, other peaks preserve their positions. Characteristic peaks of $\gamma$ phase in the third step nanoribbons are located at the same peak positions of the first step microribbons. 

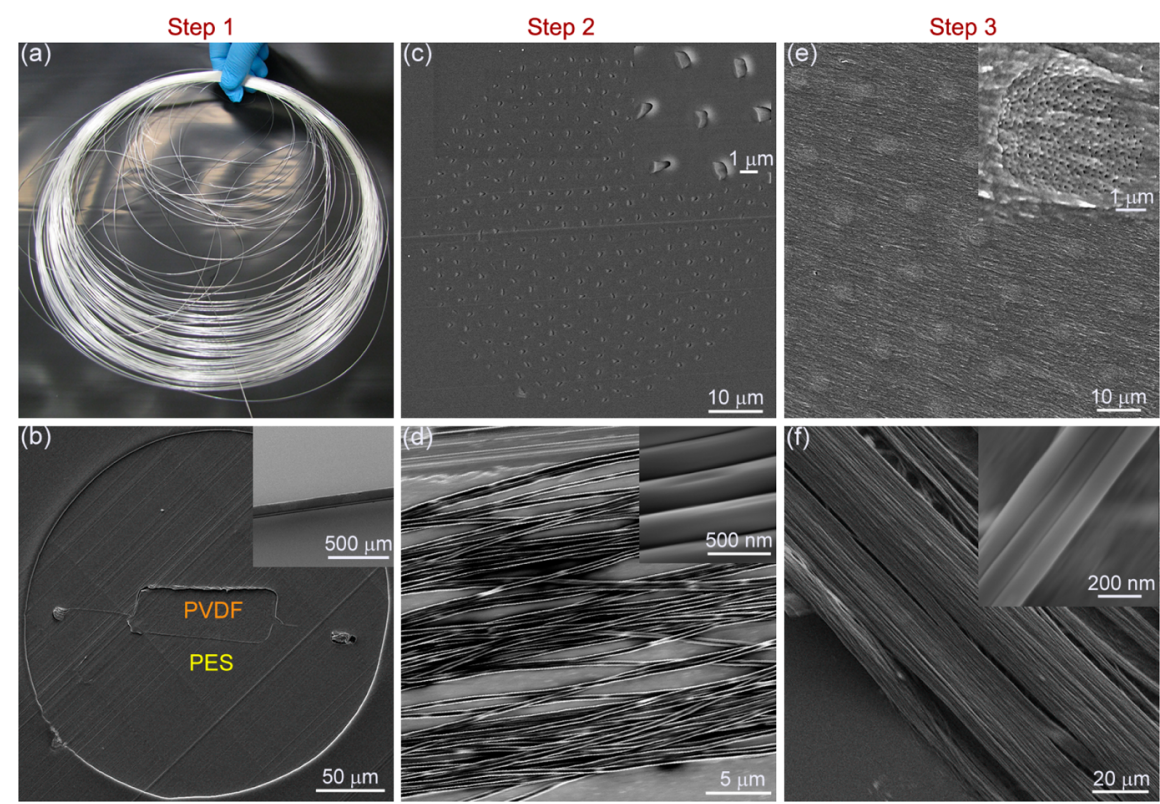

Figure 2. SEM micrographs of PVDF micro- and nanoribbons produced by using iterative size reduction technique in each drawing step. (a) Photograph of tens of meters long PDVF nanoribbon array embedded in PES cladding. (b) Cross sectional image of first step PVDF microribbon in the PES cladding. Inset: Free-standing PVDF microribbon obtained by etching PES cladding using chemical etchants. (c) Cross sectional image of second step fiber with $\sim \mathbf{4 0 0}$ nanoribbons. Inset: Close-up image of nanoribbons. (d) Lateral image of second step nanoribbons. Inset: Close-up image of aligned nanoribbons. (e) Cross sectional image of third step nanoribbon bundles in fiber. Close-up image of a single bundle. (f) SEM of third step nanoribbons extracted out of their cladding. Inset: Close-up image of aligned nanoribbons.

Broadening in the $\gamma$ phase peaks and drastic fall in $2 \theta=$ $26.8^{\circ}$ peak intensity indicate a slight decrease in amount of $\gamma$ phase in step 2 and 3.

Another tool that we used for analyzing and confirming the phase distribution in nanoribbons is ATR-FTIR. Absorption band characteristics of $\alpha, \beta$, and $\gamma$ phases of PVDF are identified as given in literature: 532, 612, 763, 796, 854, 870, 974, 1146, 1210,1383 , and $1423 \mathrm{~cm}^{\circ}$ for $\alpha ; 510,840,1279,1286$, $1431 \mathrm{~cm}^{\circ}$ for $\beta ; 812,833,838,885$, and $1234 \mathrm{~cm}^{-1}$ bands for $\gamma$. However, most of the absorption bands are superimposed for $\beta$ and $\gamma$ phases hindering the phase discrimination. ${ }^{21}$ Overlapping peak at 840, 1279, and $1286 \mathrm{~cm}^{-1}$ with $\gamma$ and $\beta$ phases can be assigned for $\gamma$ phase as long as no $\beta$ peak is identified using XRD technique for micro- and nanoribbons. Figure $3 c$ represents ATR-FTIR absorption spectra for PVDF nanoribbons produced in all steps. Fraction of the $\gamma$ phase is calculated using the following equation:

$$
F(\gamma)=\frac{X_{\gamma}}{X_{\alpha}+X_{\gamma}} \times 100=\frac{A_{\gamma}}{\left(K_{\gamma} / K_{\alpha}\right) A_{\alpha}+A_{\gamma}} \times 100
$$

where the $X_{\gamma}$ and $X_{\alpha}$ are degree of crystallinity, $A_{\gamma}$ and $A_{\alpha}$ are measured absorbance intensity, $K_{\gamma}$ and $K_{\alpha}$ are wavelength dependent absorption coefficient and $F_{\gamma}$ is the percentage of $\gamma$ phase. We calculated $\gamma$ percentage for $763 \mathrm{~cm}^{-1} \alpha$ peak and $833 \mathrm{~cm}^{-1} \gamma$ peak using corresponding absorbance coefficients $K_{\gamma}=0.150$ $\mu \mathrm{m}^{-1}$ and $K_{\alpha}=0.365 \mu \mathrm{m}^{-1}$ (Beer - Lambert Law), respectively. ${ }^{20,21} \gamma$ phase percentage in step 1 microribbons is $76 \%$, whereas step 2 and step 3 nanoribbons $\gamma$ phase percentage decreased to $72 \%$ as a result of diminishing shear force on nanoribbons exposed to heat retreatment with smaller cross sectional areas. ${ }^{48}$

Because of phase transformation into $\gamma$ phase occurs at high temperatures, stability of the $\gamma$ phase at harsh conditions needs to be investigated. We realized from sequential heat treatments to PVDF nanoribbons that $\gamma$ phase PVDF produced by iterative fiber drawing technique is quite stable at high temperatures. Nanoribbons extracted out of the PES cladding are annealed at several different temperatures up to $175^{\circ} \mathrm{C}$, and it is evident from XRD peaks that there is no significant change in $\gamma$ phase content (Figure $3 \mathrm{~d}$ ). It is clearly observed from the peaks at $2 \theta=18.6^{\circ}, 20.1^{\circ}$ and $26.8^{\circ}$, $\gamma$ phase still exists at elevated temperature. Regarding broadening and decreasing intensity of XRD peaks, we observed that crystallinity is slightly diminished due to effect of high temperature.

Structural changes due to temperature and induced stress during the fiber drawing is investigated by $a b$ initio calculations, which confirm that there is a phase transition trend from $\alpha$ and $\beta$ forms to $\gamma$ form under tensile and compressive strain (Figure $3 \mathrm{e}-\mathrm{g}$, Figure $\mathrm{S7}$ and Video $\mathrm{S} 3(\mathrm{SI})$ ). The temperature in the simulation is set to $470 \mathrm{~K}$, which corresponds to PVDF core temperature during drawing process (Figure $\mathrm{S} 8(\mathrm{SI})$ ) and which also is well above the melting temperature of PVDF. For comparison and to understand the effect of temperature, the calculations are also performed at $0 \mathrm{~K}$ (which actually corresponds to the case where temperature effects are excluded in ab initio simulations to 
(a)

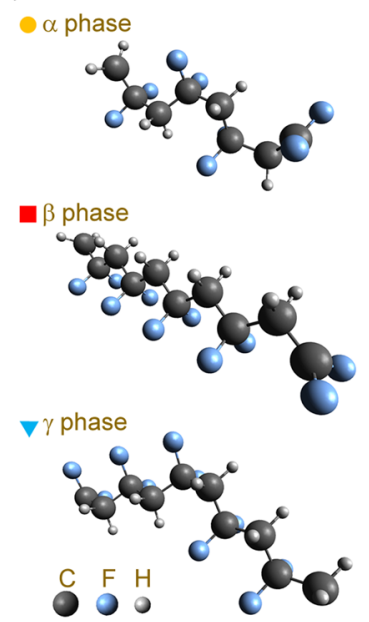

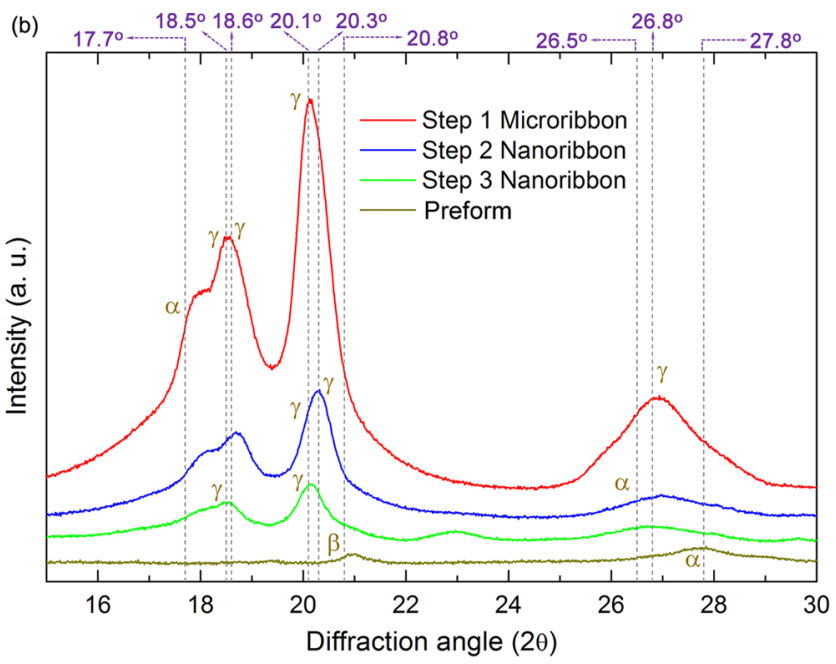

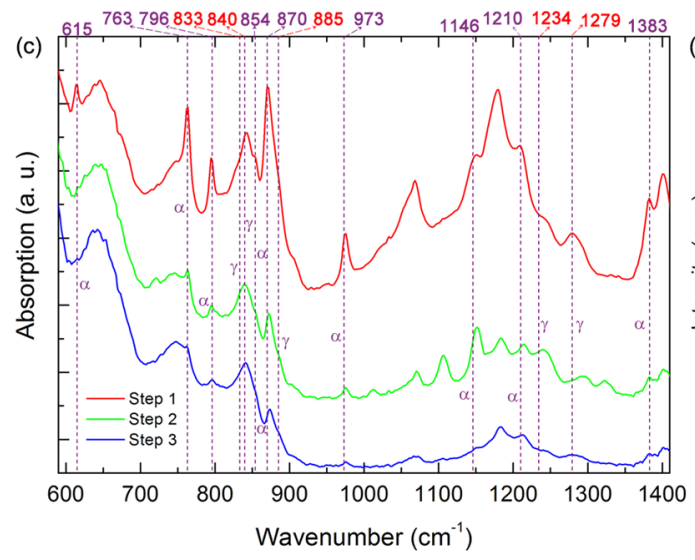

(e)

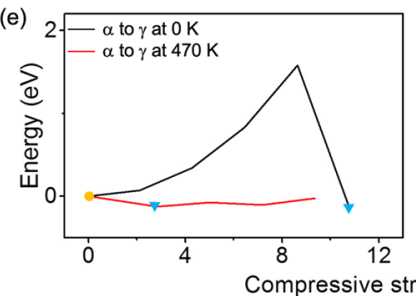

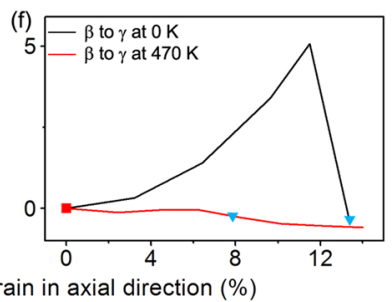

$18.6^{\circ} 20.1^{\circ}$

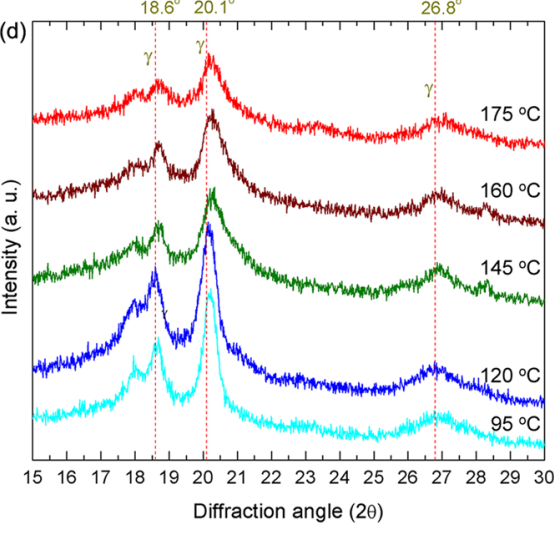

Figure 3. XRD and $a b$ initio simulation results representing $\gamma$ phase transition from PVDF slab via fiber drawing process. (a) PVDF has three main forms, which are known as nonpolar $\alpha$ phase, polar $\beta$ and $\gamma$ phases. (b) XRD data of PVDF slab extracted from a preform, microribbon and nanoribbon are taken after removing the PES cladding. Peaks observed at $18.5^{\circ}$ and $18.6^{\circ}$, $20.1^{\circ}$ and $20.3^{\circ}, 26.8^{\circ}$ correspond to the planes of $\gamma$ polar form (020), (002)/(110) and (022), respectively. $\alpha$ phase peaks at $17.7^{\circ} 26.5^{\circ}$ and $27.8^{\circ}$ are collected from (100), (021) and (111) planes. Peak at $20.8^{\circ}$ is the only $\beta$ peak observed in the slab. Spontaneous polar form ( $\gamma$ phase) is achieved after the thermal size reduction in all fiber drawing steps. (c) ATR-FTIR peaks from the first, second and third step PVDF microribbons and nanoribbons representing $\alpha$ and $\gamma$ phases. 833, 840, 885, 1234, 1279 , and $1286 \mathrm{~cm}^{-1}$ are characteristics of the $\gamma$ phase PVDF. $615,763,796,854,870,973,1146,1210$, and $1383 \mathrm{~cm}^{-1}$ are the FTIR peaks of $\alpha$ phase PVDF. Fraction of the $\gamma$ phase is $74 \%$ in first step microribbons whereas it decreases $72 \%$ in third and second step nanoribbons. (d) XRD patterns showing the characteristic peaks of the second step PVDF nanoribbons at different annealing temperatures. Although the temperature is increased above the melting point of PVDF, dominance of $\gamma$ phase is obvious from the peaks at $18.6^{\circ}, 20.1^{\circ}$ and $26.8^{\circ}$ positions. (e) Transition from $\alpha$ (yellow circle) to $\gamma$ phase (blue triangle), (f) transition from $\beta$ (red square) to $\gamma$ phase with compressive strain and (g) transition from $\alpha$ to $\beta$ phase with tensile strain on the unit cell with 6 monomers of PVDF in axial direction are simulated considering fiber drawing parameters (high temperature and shear stress) using $a b$ initio calculations. $0 \mathrm{~K}$ results are corresponding to the case where temperature effects are excluded.

obtain ground state properties). The applied force in the fiber drawing axis causes stretching in the same direction but compression in the perpendicular directions. As the orientation of the molecules with respect to fiber drawing axis in the bulk PVDF can vary, all strain components that occurred during the fiber drawing should be taken into account in the $a b$ initio model in which we considered tensile and compressive strains in the system as lattice stretching and lattice compression, respectively. The results are summarized in 
Figure $3 e-g$, where yellow circle, red square and blue triangle represent the molecular chains in Figure 3a. When compressive strain is applied in axial direction of $\alpha$ and $\beta$ form PVDF, a transformation from $\alpha$ to imperfect $\gamma$ phase with longer nonpolar parts, and a transformation from $\beta$ to ideal $\gamma$ phase are clearly observed at both 0 and $470 \mathrm{~K}$. Although a very high activation energy of 1.6 and $5.1 \mathrm{eV}$, which practically makes the transformation impossible, is required at $0 \mathrm{~K}$ for the transition from $\alpha$ and $\beta$ to $\gamma$ phase, respectively, the same phase change phenomena occur with almost no energy barrier at $470 \mathrm{~K}$. The required strain for phase transformation is significantly reduced with temperature as well. While transformations from $\alpha$ to $\gamma$ occurs at $10.8 \%$ and $\beta$ to $\gamma$ at $13.4 \%$ compressive strain at $0 \mathrm{~K}$, the same transformations are observed at 3 and $8 \%$ at $470 \mathrm{~K}$. The similar trends are observed at higher temperatures with a small amount reduction in the required strain (Figure S9 $(\mathrm{SI})$ ). These results indicate that the temperature above the melting point of PVDF during the fiber drawing process enables the phase transformation from other phases to $\gamma$ phase by decreasing the required compressive strain and energy barrier. The peaks obtained at $2 \theta=17.7^{\circ}, 26.5^{\circ}$ and $27.8^{\circ}$ from XRD data, which correspond to $\alpha$ phase PVDF as shown in Figure $3 \mathrm{~b}$, can be explained by imperfect transformation from $\alpha$ to $\gamma$ phase. In a similar manner, tensile strain in axial direction is also applied. A direct phase transition from $\alpha$ to $\beta$ phase is favored at $0 \mathrm{~K}$ when strain exceeds $14.6 \%$, and the required activation energy is $2.1 \mathrm{eV}$. Interestingly, when the temperature is elevated up to $470 \mathrm{~K}$ or more, instead of direct transition from $\alpha$ to $\beta$ phase, $\gamma$ phase appears in the first place at $2.2 \%$ transforming into a perfect $\gamma$ phase at $4.4 \%$ of tensile strain with an energy barrier of $0.07 \mathrm{eV}$. If strain is further increased and reaches $13.1 \%$, imperfect $\beta$ phase can be obtained with an energy barrier of $1.0 \mathrm{eV}$ (the imperfection of the $\beta$ phase can be due to the requirement of polarization process for the transformation $\left.{ }^{15}\right)$. Therefore, simulation results show that energy barrier for $\alpha$ to $\gamma$ transition $(0.07 \mathrm{eV}$, blue triangle in Figure $3 \mathrm{~g}$ ) under tension is lower than $\alpha$ to $\beta$ transition ( $1 \mathrm{eV}$, red square in Figure $3 \mathrm{~g}$ ) under tension.

Electrical characterizations such as piezoelectric displacement and ferroelectric hysteresis curve measurements are performed for PVDF nanoribbons. Utilizing a (Radiant Premier II) piezoelectric evaluation system along with an AFM instrument simultaneously functioning as a high precision displacement sensor and a tool for electrical coupling to nanoscale surfaces (Figure S10 (SI)), a large average effective piezoelectric coefficient $\left(d_{33}=-58.5 \mathrm{pm} / \mathrm{V}\right)$ is measured from $80 \mathrm{~nm}$ thick, $180 \mathrm{~nm}$ wide single PVDF nanoribbons isolated from an as-produced bundle (Figure 4a). As the exact shape and the size of the tip is unknown, we can only represent a ferroelectric hysteresis curve (polarization vs
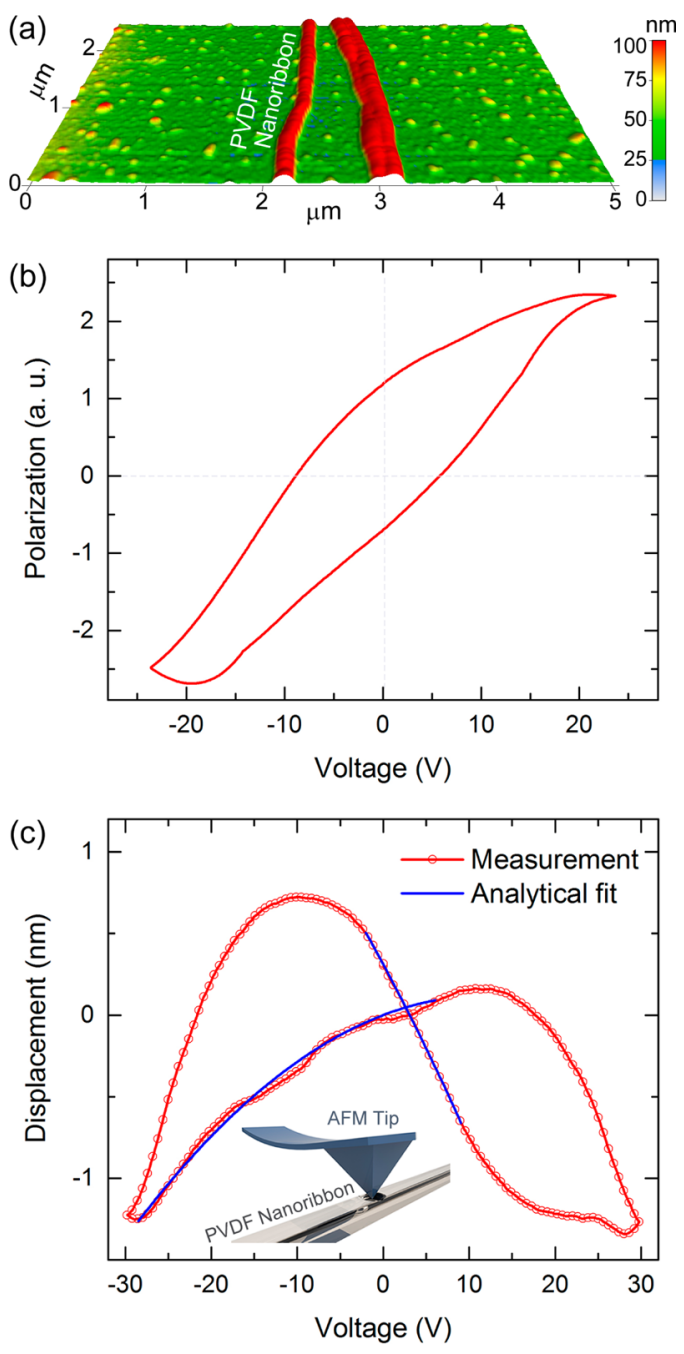

Figure 4. (a) AFM topography of $80 \mathrm{~nm}$ thick and $180 \mathrm{~nm}$ wide single and double nanoribbons on a metal coated substrate. (b) Hysteresis loop of as produced single nanoribbons at $25 \mathrm{~V}$ and $100 \mathrm{~Hz}$, which represents the spontaneous electric polarization. (c) Displacement-voltage hysteresis loop taken by an AFM and piezoelectric evaluation system from a single PVDF nanoribbon. Inverse butterfly loop is a characteristic result of the negative $d_{33}$ piezoelectric coefficient. Total measured displacement is a function of $E$ and $E^{2}$. Since the applied electric field and displacement are known, $d_{33}$ and $Q$ values can be calculated in least-squares sense. Data fitting is executed by using 370 data points.

voltage) in arbitrary polarization units. But still remnant polarization can clearly be observed (Figure 4b). Since PVDF is a multiferroic material and piezoresponse characterization of nanoscale piezoelectric materials is challenging, characterization of PVDF nanoribbons is inherently multiphysical problem that requires considering internal and external variables such as local temperature changes, electrostriction, pyroelectricity, ferroelasticity, electrostatic effects, indentation regime, applied electrical potential, contact (AFM Tip) sliding and drifting effects. Piezoelectric coefficient measurements using AFM and a piezoelectric evaluation system (virtual ground mode) can be well understood in three 
sequential stages: sample preparation and mechanical contact with AFM tip, recording piezoelectric response, analyzing the acquired signals for calculating pure piezoelectric displacement. The ribbon structure provides convenience for piezoelectric measurements with AFM since a more conformal contact between the bottom of the nanoribbon and the conductive surface of the substrate diminishes the sliding and drifting adversities during measurements. Before local piezoelectric characterization, we operated a noncontact mode AFM surface imaging for locating a PVDF single nanoribbon among dispersed nanoribbons on a $60 \mathrm{~nm}$ silver coated silicon wafer. After a mechanical contact between AFM tip and the surface of the nanoribbon is accomplished in contact mode AFM, we conducted displacement-voltage $(D-V)$ measurements applying $10 \mathrm{~ms}$ bipolar triangular voltage pulses between the AFM tip (electrical potential) and the metal coating of the substrate (ground). During piezoelectric measurements, we kept AFM control loop off and recorded piezoelectric displacements in a very short time scale compared to that of AFM tip drift. In addition, we used multiple deflection measurements from each local contact surface and calculated the average $D-V$ curve in order to analytically cancel drifting effects and calculate a more accurate piezoelectric coefficient. AFM is one of the most precise deflection sensors that can dynamically detect the change in the thickness of PVDF nanoribbons according to alternating electric potential. However, many artifacts can occur related to applied electric field and contact indentation regime during piezoelectric measurements at nanoscales. We used experimental and analytical approaches in order to eliminate such effects and analyze the origin of the large displacement in PVDF nanoribbons. First, electrostatic forces can dislocate AFM tip in nanoscale distances. This effect can be simply eliminated using a stiffer $(k=40 \mathrm{~N} / \mathrm{m}$ and $f=300 \mathrm{kHz}$ ) AFM tip. Measured signal from AFM deflection corresponds to the change in the thickness of PVDF nanoribbon because, in principle, AFM tip follows the surface motions of the sample. From the mechanics of materials perspective, we can calculate the total strain $(s=\Delta t / t)$ in PVDF nanoribbons, where $t$ is the thickness of the nanoribbons and $\Delta t$ is the measured change in the thickness. The measured strain is not a pure piezoelectric deflection, but rather a sum of strain components caused by electrostriction, thermal effects and applied pressure in the direction of the electric field.

$$
\begin{gathered}
s=s_{\text {piezoelectric }}+s_{\text {electrostriction }}+s_{\text {thermal }}+s_{\text {pressure }} \\
s=d_{33} E-Q E^{2}+\lambda \Delta T+e_{33} \sigma_{33}
\end{gathered}
$$

where $d_{33}$ is the piezoelectric coefficient, $E$ is the electric field, $Q$ is the electrostriction coefficient, $\lambda$ is the thermal expansion coefficient, $\Delta T$ is the change in the temperature, $e_{33}$ is the elastic coefficient and $\sigma_{33}$ is the stress. The pressure induced strain related to indentation regime or AFM tip can trigger ferroelastic motions in PVDF nanoribbons, unless the indentation force is very small and constant. Since we need to apply a voltage to our conductive tip, it is required to make a mechanical contact with the surface of the nanoribbons. A COMSOL Multiphysics simulation is designed to analyze the deformation of contact region during piezoelectric measurements (Figure S11 (SI)). The simulation results show that applying a $-60 \mathrm{nN}$ indentation force on the $80 \mathrm{~nm}$ thick PVDF nanoribbon, which corresponds to $15 \mathrm{~nm}$ static deflection in AFM cantilever, triggers maximum $0.3 \mathrm{~nm}$ elastic deformation on the surface with an AFM tip diameter of $10 \mathrm{~nm}$. Therefore, AFM tip is guaranteed to be in constant mechanical contact with the nanoribbon surface during measurements, due to AFM tip deflection range is higher than the total piezoelectric displacement measured. Dilatation of PVDF nanoribbons and pyroelectric effects can also be ignored, because all measurements are conducted at constant room temperature. In addition, to consider the local temperature changes caused by joule heating, we modeled the change in the PVDF nanoribbon temperature as a function of the electric potential (Figure S12 (SI)). Joule heating is proportional to $i^{2}$ and $R$, where $i$ is the traveling current across the PVDF thickness and $R$ is the resistance. Since resulting current is very small, there is no change in the temperature caused by joule heating. Experimentally eliminating the pressure and temperature dependent strain components results in eq 4.

$$
s=d_{33} E-Q E^{2}
$$

Inserting the measured data (displacement vs applied field) in eq 4 , an overdetermined system of equations for two unknowns $\left(d_{33}\right.$ and $Q$ ) is obtained, which can be solved in least-squares sense as shown in the Supporting Information. Results are perfectly fitted to the measured curve (Figure 4c). We calculated that electrostriction and piezoelectric coefficient of $\gamma$ phase PVDF nanoribbons are $Q=-67.8 \times 10^{-9} \mathrm{pm}^{2} / \mathrm{N}^{2}$ and $d_{33}=-58.5 \mathrm{pm} / \mathrm{V}$. In the range of a maximum $\pm 10 \mathrm{~V}$ applied electric potential differance, $87.4 \%$ (585.3 pm) and $12.6 \%$ ( $84.47 \mathrm{pm}$ ) of the deflection result from pure piezoelectric effect and electrostriction, respectively. The measured average effective piezoelectric coefficient of $\gamma$ phase PVDF nanoribbons is higher than the reported values of $\beta$ phase $\left(d_{33}=-30 \mathrm{pm} / \mathrm{V}\right)$ and $\gamma$ phase $\left(d_{33}=-7 \mathrm{pm} / \mathrm{V}\right)$ PVDF thin films, ${ }^{15,21}$ and on same order of magnitude with $\beta$ phase $\left(d_{33}=-57.6 \mathrm{pm} / \mathrm{V}\right)$ PVDF nanowires, which are characterized in a similar manner using AFM. ${ }^{49}$ Unlike the nanoribbons, the effective $d_{33}$ coefficient for thin films is expected to be reduced, due to surface clamping boundary conditions. $^{50}$ The reverse form of $D-V$ hysteresis loop (Butterfly Loop) is due to the negative value of 
(a)
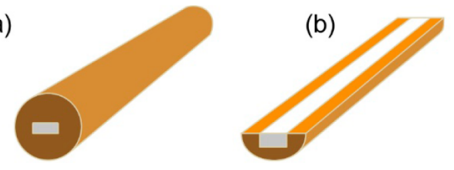

(e)

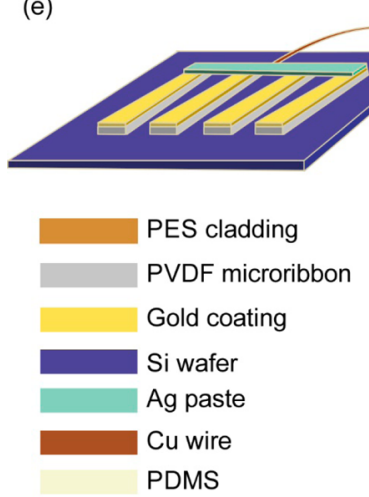

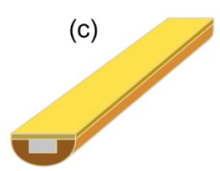

(d)
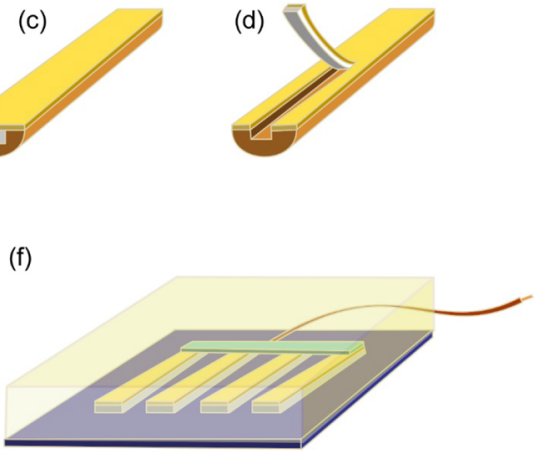

(g)

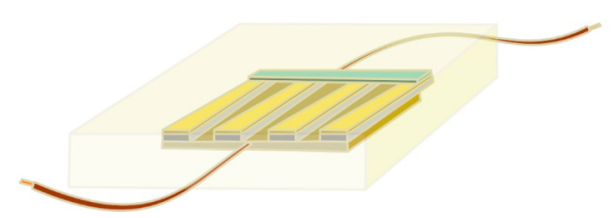

Figure 5. Fabrication process for the device produced using first step microribbons. (a) A $50 \mu \mathrm{m}$ thick microribbon embedded single fiber is selected. (b) The fiber is longitudinally divided in two pieces. (c) The surface of the piece with the ribbon trapped is coated $50 \mathrm{~nm}$ gold. (d) After carving the PVDF microribbon out of the cladding, it is cut in equal pieces. (e) One side coated microribbons are aligned on a silicon substrate. Next, Ag paste and Cu wire electrodes are attached on the gold coated surface of the microribbons. (f) The structure is transferred onto a PDMS layer. (g) The back side of the PVDF microribbons are also coated with $50 \mathrm{~nm}$ gold, and the whole device is embedded in PDMS.

$d_{33}$ coefficient (Figure 4c). The general relation between piezoelectric coefficients of PVDF is $d_{33} \geq d_{31}>$ $d_{32}>0 .^{30,48}$ In order to confirm the measurent technique, we conducted the same measurement on $\alpha$ phase commercial PVDF thin film with $60 \mu \mathrm{m}$ thinckness, $25 \mu \mathrm{m}^{2}$ surface area, and we observed no significant deflection as expected.

We developed two devices with different geometries using PVDF micro- and nanoribbons produced by thermal size reduction technique. In the first device, a first step fiber is longitudinally divided in two halves without damaging the PVDF microribbon and one face of the microribbon PVDF is uncovered. $50 \mathrm{~nm}$ gold is sputtered on the open surface of the microribbon. After mechanically removing the remaining part of PES cladding, the microribbon is cut in smaller pieces and aligned on a silicon substrate, so that the gold deposited faces are on the top. A contact pad is attached to the gold coated surface of $50 \mu \mathrm{m}$ thick PVDF microribbons, and the structure is transferred onto a polydimethylsiloxane (PDMS) layer which avoids the short circuit of the device and maintains the alignment of the microribbons. Subsequently, the other surfaces of the microribbons are also coated with $50 \mathrm{~nm}$ thick gold, and whole device is embedded in PDMS (Figure $5 \mathrm{a}-\mathrm{g}$ ). The second device with a different structure convenient for nanoribbons is fabricated using $300 \mathrm{~nm}$ PVDF ribbons, which are extracted out of the PES cladding using DCM. The both side of the nanoribbon bundles are coated with sputtering of $50 \mathrm{~nm}$ gold film using a shadow mask (Figure S13 (SI)). Misalignment of nanoribbons is expected to be reduced output voltage and current of the device. Effective area of the devices fabricated using microribbons (Figure 6a) and nanoribbons (Figure $6 \mathrm{~b}$ ) are $100 \mathrm{~mm}^{2}$ and $20 \mathrm{~mm}^{2}$, respectively. Characterizations of the devices are carried out with an external load capacitance $\left(C_{\mathrm{L}}=16 \mathrm{pF}\right)$ and resistance $\left(R_{\mathrm{L}}=10 \mathrm{M} \Omega\right)$. An equivalent circuit (Figure $6 \mathrm{c}$ ) for piezoelectric devices can be represented by a parallel RC circuit containing a charge source $(\mathrm{q})$, a resistor $\left(R_{0}\right)$, capacitor $\left(C_{0}\right)$. From DC and impedance measurements (Figure S14 $(\mathrm{SI})$ ), internal resistor $\left(R_{0}\right)$ and capacitor $\left(C_{0}\right)$ for the first and second device are calculated to be $R_{0}=$ $100 \mathrm{G} \Omega$ and $C_{0}=3.4 \mathrm{pF}, R_{0}=40 \mathrm{G} \Omega$ and $C_{0}=4.6 \mathrm{pF}$, respectively.

Because of PVDF dipoles oriented perpendicular to the fiber axis, when a force is applied vertical to the fiber axis, a positive piezoelectric potential is produced and collected on positive electrode. The same phenomenon occurs vice versa during the releasing. Output voltages and currents of the devices are recorded under quasi-periodic tapping forces (Video S4 (SI)). The output voltage and current are related to the magnitude and period of tapping force. The typical output values of the device fabricated using microribbons is $6 \mathrm{~V}$ and $3 \mu \mathrm{A}$ (Figure $6 \mathrm{~d}, \mathrm{e}$ ), and the typical output values of the device produced using nanoribbons is $40 \mathrm{~V}$ and $6 \mu \mathrm{A}$ (Figure $6 \mathrm{f}, \mathrm{g}$ ). Although microribbons have $4 \%$ higher amount of polar phase content, charge collected from the device built with nanoribbons is approximately 9 fold higher due to greater contact surface area ( 2 orders of magnitude higher) and better charge collection efficiency of nanoribbons. Maximum output ( $60 \mathrm{~V}$ and $10 \mu \mathrm{A}$ for the nanoribbons, $7 \mathrm{~V}$ and 
(a)

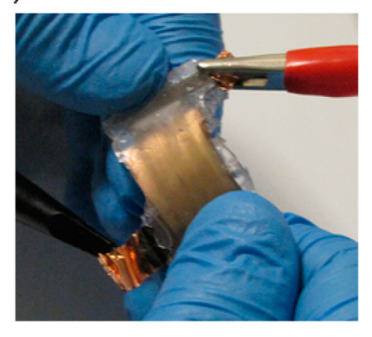

(b)

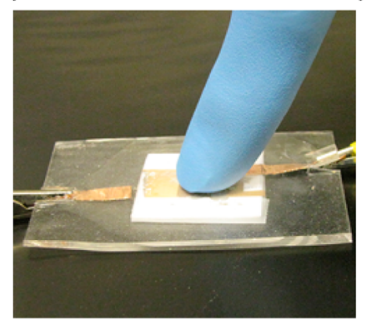

(c)

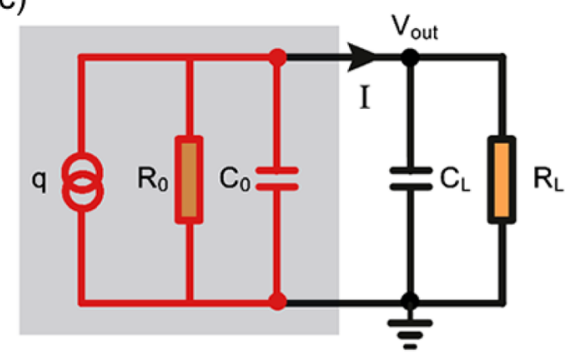

(d)

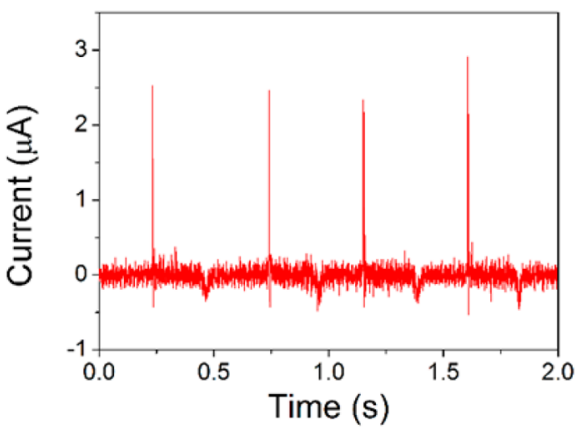

(f)

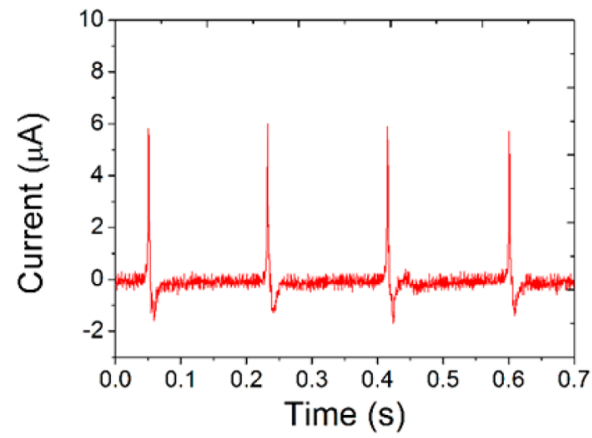

(e)

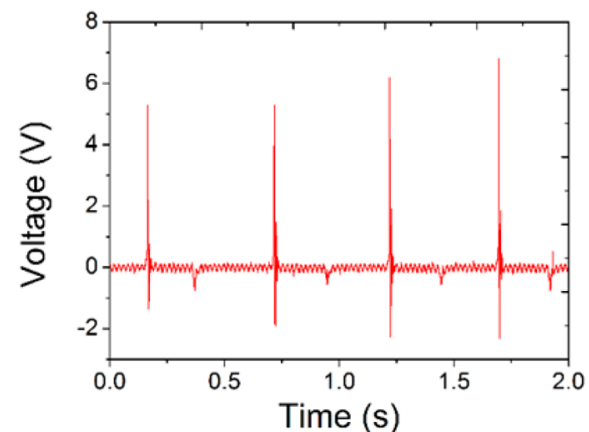

(g)

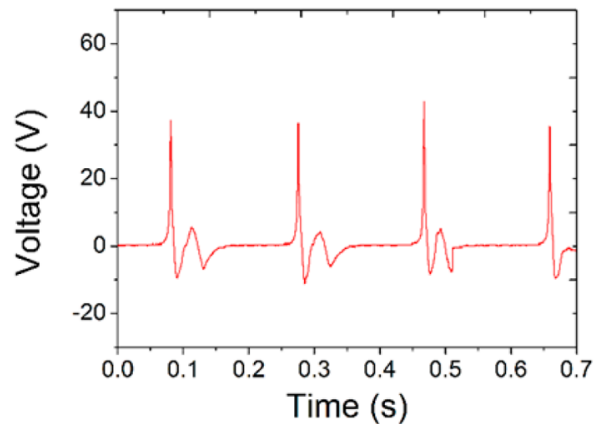

Figure 6. Electrical characterization of the piezoelectric devices. (a) Devices fabricated using $50 \mu \mathrm{m}$ thick microribbons and (b) $300 \mathrm{~nm}$ thick nanoribbons. (c) A piezoelectric device can be modeled as a parallel RC circuit containing a charge source $(q)$, resistor $\left(R_{0}\right)$ and capacitor $\left(C_{0}\right)$, which is represented by red lines on gray background. The remaining part of the circuit $\left(C_{\mathrm{L}}\right.$ and $R_{\mathrm{L}}$ ) belongs to an external load. (d) Current and (e) voltage output measurements of the device produced using $50 \mu \mathrm{m}$ thick PVDF microribbons. (f) Current and (g) voltage output measurements of the device produced using $300 \mathrm{~nm}$ thick PVDF nanoribbons.

$3 \mu \mathrm{A}$ for the microribbons) of the piezoelectric devices can be seen from a broader range of the electrical measurements given in Figure S15 (SI). Besides, peak output power densities of first and second devices are 5.25 and $750 \mu \mathrm{W} / \mathrm{cm}^{2}$, which prove that efficiency of the nanoribbons is much higher. We built another device using nonpiezoelectric amorphous $\mathrm{As}_{2} \mathrm{Se}_{3}$ nanowires (150 nm in diameter) produced by thermal fiber drawing technique in order to confirm piezoelectric effect observed in our devices (Figure S16 (SI)). There is no response observed except noise from the nonpiezoelectric device, despite the device produced using PVDF nanoribbons can response even for small tapping forces.

\section{CONCLUSION}

We introduced a novel top-down solvent free method for the production of dominantly $\gamma$ phase onedimensional PVDF nanostructures. PVDF micro- and nanoribbons ranging from $100 \mu \mathrm{m}$ to $5 \mathrm{~nm}$ in thickness are produced by using iterative fiber drawing technique in three drawing steps. As-produced nanoribbons have an ultrahigh aspect ratio $(\mathrm{km} / \mathrm{nm})$, good uniformity with cross sectional ribbon geometry, high thermal stability, and high piezoelectric properties. XRD and FTIR results reveal that the $\gamma$ phase is the dominant phase $(72 \%)$ in all nanoribbons. The shear stress and high temperature applied during fiber drawing process favor the polar $\gamma$ phase transition. Simulating the fiber drawing process using $a b$ initio calculations, we confirmed that transformation into $\gamma$ phase from other phases at elevated temperature above the melting point of PVDF is favorable. Moreover, PVDF nanoribbons are annealed at several different temperatures up to $175^{\circ} \mathrm{C}$, and XRD results show that $\gamma$ phase still exists after annealing. Piezoelectric characterizations of single $80 \mathrm{~nm}$ thick nanoribbons 
are accomplished by exploiting a piezo evaluation system and an AFM Instrument as a nanoscale probing tool. To our knowledge, we report the highest effective piezoelectric coefficient $\left(d_{33}=-58.5 \mathrm{pm} / \mathrm{V}\right)$ measured from a $\gamma$ phase PVDF single nanoribbon. In addition, we built two proof of principle devices using $50 \mu \mathrm{m}$ and $300 \mathrm{~nm}$ ribbons for the purpose of mechanical energy harvesting and tapping sensor application. Short circuit peak voltage and peak short circuit current outputs of the devices are measured up to $60 \mathrm{~V}$ and $10 \mu \mathrm{A}$. Our devices can be used with an energy harvesting circuit (rectifier and storage capacitor with a switching element) in low power requiring applications. Because of the polymer encapsulation, nanoribbon arrays can be transferred on any substrate and chemically extracted out of their polymer jacket for further device integration such as piezoelectric nanoribbons on interdigitated metal electrodes. Iterative fiber drawing technique holds a huge potential due to the capacity for multimaterial (metal-piezoelectric-dielectric) coprocessing in order to produce building blocks for large area, flexible, lightweight, long endurance, costeffective piezoelectric devices such as artificial muscle and skin, smart textiles, and energy generators.

\section{EXPERIMENTAL SECTION}

Preform Fabrication. Iterative fiber drawing technique requires the use of composite (multimaterial) preforms. We produced one PVDF and three PES preforms. PVDF films (Ajedium Films - Solvay Plastics) with a thickness of $60 \mu \mathrm{m}$ are used for the fabrication of PVDF preform, which is $30 \mathrm{~mm}$ in diameter and $20 \mathrm{~cm}$ in length. In degassing and consolidation process, the preform is held in a vacuum oven for $4 \mathrm{~h}$ at $140{ }^{\circ} \mathrm{C}$ under $2 \times 10^{-2}$ Torr pressure and consolidated for $30 \mathrm{~min}$ at $180^{\circ} \mathrm{C}$ under $2 \times 10^{-2}$ Torr vacuum pressure. Finally a slab $3 \mathrm{~mm}$ in thickness, $10 \mathrm{~mm}$ in width and $10 \mathrm{~cm}$ in length is mechanically extracted from the preform to be inserted into the first step PES preform. PES preforms are produced using one side polished $100 \mu \mathrm{m}$ thick PES films (Ajedium Films). PES preforms are $35 \mathrm{~mm}$ in diameter, and $25 \mathrm{~cm}$ in length and inner diameters of preforms are $3 \mathrm{~mm}$. In degassing and consolidation process, preforms are held in a vacuum oven for $4 \mathrm{~h}$ at $180^{\circ} \mathrm{C}$ under $2 \times 10^{-2}$ Torr pressure and consolidated for $35 \mathrm{~min}$ at $255^{\circ} \mathrm{C}$ under $2 \times 10^{-2}$ Torr vacuum pressure. The first step PES preform is different from the second and third step preforms: To insert the PVDF slab inside the first step PES preform, we splited the preform in two parts and machined both halves.

Iterative Fiber Drawing Process. Fiber fabrication process consists of three consecutive fiber drawing steps. $3 \mathrm{MPa}$ tensile stress is applied during fiber drawing through the fiber drawing axis. The temperature used in fiber drawing is $285^{\circ} \mathrm{C}$, which is well above the glass transition temperature and melting temperature of PVDF. Optimized preform feeding speed is found to be $8 \mathrm{~mm} / \mathrm{sec}$. The thickness of the ribbons ranges between 400 to $10 \mu \mathrm{m}$ in the first step, 300 to $50 \mathrm{~nm}$ in the second step and 50 to $5 \mathrm{~nm}$ in the third step. Approximately 400 fibers are used in the second and third steps.

Morphological and Chemical Characterizations. SEM images are taken using FEI Quanta 200 FEG electron microscopy with low voltage-high contrast detector (vDS) under a vacuum at $6 \times$ $10^{-4} \mathrm{~Pa}$ chamber pressure. We used $5 \mathrm{kV}$ acceleration and $4 \mathrm{kV}$ deceleration (bias) beam voltage within $4 \mathrm{~mm}$ working distance. Lateral PVDF nanoribbon images are taken after the PES cladding of fibers are etched using DCM and $4 \mathrm{~nm} \mathrm{Au} / \mathrm{Pd}$ conductive film is sputtered on to the surface. To obtain cross sectional images, a special sample preparation process is used. First, fibers are embedded in a resin (Technovit 7100). Using a Leica EMUC6 - EMFC6 Ultramicrotome with a dimond knife, fiber cross sections are smoothed after the cryogenic chamber of the ultramicrotome is cooled down to $-125^{\circ} \mathrm{C}$ using liquid nitrogen. Before SEM images are taken, $4 \mathrm{~nm} \mathrm{Au} / \mathrm{Pd}$ conductive film is coated on the smoothed surfaces. To enhance the electrical conduction, each sample is painted using silver paste. XRD patterns of PVDF ribbons are taken by Pananalytical X'pert Pro XRD with a diffraction angle $2 \theta$ scanned between 5 to 75 degrees using a step size of 0.01 degrees and a dwell time of 800 s per step. Thermo Scientific Nicolet 6700 FTIR with an ATR attachment is used for molecular confirmations. FTIR reflection data is obtained with a wavelength scan resolution of $0.482 \mathrm{~cm}^{-1}$ and a total of 256 scan steps.
$\boldsymbol{A} \boldsymbol{b}$ Initio Calculations. In order to simulate the phase transformation in iterative fiber drawing process and investigate the effect of temperature, $a b$ initio calculations based on density functional theory ${ }^{51,52}$ are carried out using Vienna $a b$ initio simulation package (VASP). ${ }^{53,54}$ To understand the effect of compressive and tensile strength, $\alpha-, \beta$, and $\gamma$-PVDF with 6 monomer chains in the unit cell is considered at temperatures equal to and higher than drawing temperature $(470 \mathrm{~K})$, which are compared with ground state results at $0 \mathrm{~K}$. Exchangecorrelation energy is expressed by the generalized gradient approximation (GGA) using PBE functional. ${ }^{55}$ The projector augmented wave (PAW) potentials are used for each element with a kinetic energy cutoff of $500 \mathrm{eV}^{56}$ All structures are relaxed with simultaneous minimization of the total energy and the interatomic forces. The convergence on the total energy and force was set to $10^{-5} \mathrm{eV}$ and $10^{-2} \mathrm{eV} / \mathrm{A}$, respectively. For high temperature calculations $a b$ initio molecular dynamics is enabled where microcanonical ensemble is simulated with $1 \mathrm{fs}$ time steps.

Piezoelectric Characterization. We used Radiant Technologies Premier II precision multiferroic piezoelectric evaluation system along with Oxford Instruments-Asylum Research MFP-3D AFM System for piezoelectric characterizations of a single PVDF nanoribbon. PES cladding of $80 \mathrm{~nm}$ thick PVDF nanoribbons are etched using DCM and nanoribbons are extracted on a $60 \mathrm{~nm}$ silver coated Si substrate. Position of a single PVDF nanowire is detected by operating a noncontact mode AFM surface imaging. We kept AFM control loop off during piezoelectric measurements. A stiff AFM tip $(k=40 \mathrm{~N} / \mathrm{m}$ and $f=$ $300 \mathrm{kHz}$ ) is used to execute accurate measurements. Polarization curve is measured at $100 \mathrm{~Hz}$ and up to $25 \mathrm{~V}$. Deflectionvoltage curve measurements are accomplished by applying 10 milisecond bipolar triangular voltage up to $30 \mathrm{~V}$. Drifting effects are eliminated by averaging multiple measurements. Pure piezoelectric coefficient, electrostriction coefficient and other effects are calculated using MATLAB 13.0 and equations are solved in least-squares sense as shown in the Supporting Information.

Finite Element Simulations. Using the software SolidWorks 2013 Simulation, three-dimensional numerical finite element method is conducted to show the distribution of normal and shear stress when a $3 \mathrm{MPa}$ triggerring stress is applied trough the fiber drawing axes for polymer materials. Effect of the gravity $\left(9.81 \mathrm{~m} / \mathrm{s}^{2}\right)$ is also taken into account. Simulation temperature is considered constant at fiber drawing temperature $\left(258{ }^{\circ} \mathrm{C}\right)$. To find out the yield characteristics in fiber drawing process, the maximum von Mises stress $\left(\sigma_{\text {vonmises }}\right)$ criterion, which is based on the von Mises-Hencky theory is appled to the system using $\sigma_{\text {vonMises }}=\left[\left(\sigma_{1}-\sigma_{2}\right)^{2}+\left(\sigma_{2}-\sigma_{3}\right)^{2}+\left(\sigma_{1}-\sigma_{3}\right)^{2} / 2\right]^{1 / 2}$ governing equation, where $\sigma_{1}, \sigma_{2}$ and $\sigma_{3}$ are the principle stresses. The Cauchy stresses, shear stress caused by applied tensile stress, strain and displacement with respect to the initial parameters are also investigated. Firictional forces due to air in the environment and free body forces are ignored. In order to be able to simulate fiber drawing, we used a linear elastic isotropic 
model type with large displacement option. Curvature based mesh used with 4 Jacobian points. Temperature distribution in fiber drawing furnace is simulated using COMSOL 4.3 heat transfer model, which follows the first rule of the thermodynamics. We ignored viscous heating and pressure work. Therefore, we concluded that $\rho C_{p} \partial T / \partial t+\rho C_{p} u \cdot \nabla T=\nabla \cdot(k \nabla T)+Q$ is our governing equation, where $C_{p}$ is the specific heat capacity, $T$ is the absolute temperature, $u$ is the velocity vector, $\rho$ is the density, $Q$ is the heating term and $t$ is time. We assumed that mass is always conserved in the furnace which means $\partial \rho / \partial t+\nabla \cdot(\rho V)=0$, where $V$ is the volume. Heat transfer interfaces of the furnace such as out flowing heat boundaries and insulating boundaries use Fourier's law of heat conduction, which means $q_{i}=-\Sigma_{j} k_{i j} \partial T / \partial x_{j}$, where $q_{i}$ is heat flux, $k_{i j}$ is anisotropic thermal conductivity tensor and $x_{j}$ is distance. This governing equation is also valid for joule heating simulations, which are performed using COMSOL 4.3 software. However, heating term is expressed with $Q=j \cdot E$ equation because joule heating is heat transfer phenomenon related to current density $(j)$ and electric field $(E)$. The linear elastic deflection that occurred due to $60 \mathrm{nN}$ indentation force is also modeled using COMSOL 4.3 software. The simulation is conducted at constant room temperature. Tip radius is chosen as $10 \mathrm{~nm}$ for all simulations. Hook's law is used in linear elastic model. Governing equation for total strain, which is a function of displacement gradient, can be expressed as $\varepsilon=1 / 2\left(\nabla d+\nabla d^{T}\right)$, equation of motion is $\nabla \cdot \sigma=F_{V}$, and strain-stress relation can be expressed as $\sigma_{i j}=C_{i j k l} \varepsilon_{k l}$, where $\varepsilon$ and $\varepsilon_{k l}$ are the strain tensors, $\nabla d$ is the gradient of the displacement and $\nabla d^{T}$ is the transpose of displacement gradient, $\sigma$ and $\sigma_{i j}$ are the Cauchy stress tensors, $F_{V}$ is the body force per unit volume and $C_{i j k l}$ is the fourth order stiffness tensor, which is a function of poisson ratio and linear elastic modulus.

Device Fabrication and Characterization. We developed two device geometries using micro- and nanoribbons with 100 and $20 \mathrm{~mm}^{2}$ effective areas, respectively. The first device is built using $50 \mu \mathrm{m}$ thick PVDF microribbons. The microribbons are mechanically extracted out of their polymer jacket and $50 \mathrm{~nm}$ gold sputtered on to the both sides as electrical contacts. The second device is fabricated using nanoribbons with $300 \mathrm{~nm}$ thickness. Polymer jacket of nanoribbons are etched using DCM, and both sides of PVDF bundles are coated with $50 \mathrm{~nm}$ gold using a shadow mask. Finally, the structures are embedded in PDMS (Sygard 184 Silicone Elastomer KIT). In the preperation of PDMS, base and curing agent are mixed using 10:1 ratio. The mixture is degassed for $1 \mathrm{~h}$ under $10^{-2}$ Torr vacuum pressure. After pouring the PDMS on PVDF ribbons devices are held under $10^{-2}$ Torr vacuum pressure at room temperature for 2 days. DC resistance of devices are measured by Keithley 2400 Source Meter. Impedance of the devices are measured using Cascade Microtech PM-5 probe station, and analytical data fitting is executed using MATLAB 13.0 software. Current and voltage output of piezoelectric devices are measured with Stanford SR-570 Current Preamplifier and Tetronix TDS-1012B Oscilloscope.

Conflict of Interest: The authors declare no competing financial interest.

Acknowledgment. We thank Murat Dere for his help during preform preparation, fiber drawing, and cross-sectioning, and Mustafa Fatih Genisel, Mustafa Urel, and Emre Karabeyoglu for useful discussions. This work was partially supported by TUBITAK under the Project Nos. 110M412 and 111A015. The research leading to these results has received funding from the European Research Council under the European Union's Seventh Framework Programme (FP/2007-2013)/ERC Grant Agreement No. 307357. M.B. acknowledges partial support from the Turkish Academy of Sciences (TUBA).

Supporting Information Available: Detailed information about process conditions for transition among $\alpha, \beta$ and $\gamma$ phases of PVDF, $a b$ initio calculations, preform preparation and consolidation process, fiber drawing process, nanoribbons size distribution, XRD and FTIR analysis, thermal stability of PVDF, experimental setup for piezoelectric characterization of PVDF nanoribbons, device fabrication and characterization. This material is available free of charge via the Internet at http://pubs.acs.org

\section{REFERENCES AND NOTES}

1. Kawai, H. Piezoelectricity of Poly(vinylidene fluoride). Jpn. J. Appl. Phys. 1969, 8, 975-976.

2. Chu, B.; Zhou, X.; Ren, B.; Neese, B.; Lin, M.; Wang, Q.; Bauer, F.; Zang, Q. M. A Dielectric Polymer with High Electric Energy Density and Fast Discharge Speed. Science 2006, 313, 334-336.

3. Nguyen, T. D.; Deshmukh, N.; Nagarah, J. M.; Kramer, T.; Purohit, P. K.; Berry, M. J.; McAlpine, M. C. Piezoelectric Nanoribbons for Monitoring Cellular Deformations. Nat. Nanotechnol. 2012, 7, 587-593.

4. Qi, Y.; Jafferis, N. T.; Lyons, K., Jr; Lee, C. M.; Ahmad, H.; McAlpine, M. C. Piezoelectric Ribbons Printed onto Rubber for Flexible Energy Conversion. Nano Lett. 2010, 10, 524528.

5. Park, K.; Xu, S.; Liu, Y.; Hwang, G. T.; Kang, S. J. L.; Wang, Z. L.; Lee, K. J. Piezoelectric $\mathrm{BaTiO}_{3}$ Thin Film Nanogenerator on Plastic Substrates. Nano Lett. 2010, 10, 4939-4943.

6. Zang, J. X.; Xiang, B.; He, Q.; Seidel, J.; Zeches, R. J.; Yu, P.; Yang, S. Y.; Wang, C. H.; Chu, Y. H.; Martin, L. W.; et al. Large Field-Induced Strains in a Lead-Free Piezoelectric Material. Nat. Nanotechnol. 2011, 6, 98-102.

7. Persano, L.; Dagdeviren, C.; Su, Y.; Zang, Y.; Girardo, S.; Pisignano, D.; Huang, Y.; Rogers, J. A. High Performance Piezoelectric Devices Based on Aligned Arrays of Nanofibers of Poly(vinylidenefluoride-co-trifluoroethylene). Nat. Commun. 2013, 4, 1633-1643.

8. Liu, Y. T.; Fung, R. F.; Wang, C. C. Precision Position Control Using Combined Piezo-VCM Actuators. Precis. Eng. 2005, 29, 411-422.

9. Mirfakhrai, T.; Madden, J. D. W.; Baughman, R. H. Polymer Artificial Muscles. Mater. Today 2007, 10, 30-38.

10. Someya, T.; Sekitani, T.; Iba, S.; Kato, Y.; Kawaguchi, H.; Sakurai, T. A Large-Area, Flexible Pressure Sensor Matrix with Organic Field-Effect Transistors for Artificial Skin Applications. Proc. Natl. Acad. Sci. U. S. A. 2004, 101, 9966-9970.

11. Bing-Huei, C.; Long, W.; Ming-Cheng, C.; Yeong-Chin, C. In Fabrication of PZT BY sol-gel method, Piezoelectricity, Acoustic Waves and Device Applications (SPAWDA), 2010 Symposium on, 10-13 Dec, 2010; pp 310-314.

12. Saito, Y.; Takao, H.; Tani, T.; Nonoyama, T.; Takatori, K.; Homma, T.; Nagaya, T.; Nakamura, M. Lead-Free Piezoceramics. Nature 2004, 432, 84-87.

13. Hattori, T.; Kanaoka, M.; Ohigashi, H. Improved Piezoelectricity in Thick Lamellar $\beta$-Form Crystals of Poly(vinylidene fluoride) Crystallized Under High Pressure. J. Appl. Phys. 1996, 79, 2016-2022.

14. Chang, C.; Tran, V. H.; Wang, J.; Fuh, T. K.; Lin, L. Direct-Write Piezoelectric Polymeric Nanogenerator with High Energy Conversion Efficiency. Nano Lett. 2010, 10, 726-731.

15. Mohammadi, B.; Yousefi, A. A.; Bellah, S. M. Effect of Tensile Strain Rate and Elongation on Crystalline Structure and Piezoelectric Properties of PVDF Thin Films. Polym. Test. 2006, 26, 42-50.

16. Gregorio, R., Jr. Determination of the $\alpha, \beta$, and $\gamma$ Crystalline Phases of Poly(vinylidene fluoride) Films Prepared at Different Conditions. J. Appl. Polym. Sci. 2006, 100, 3272-3279.

17. Li, M.; Wondergem, H. J.; Spijkman, M. J.; Asadi, K.; Katsouras, I.; Blom, P. W. M.; de Leeuw, D. M. Revisiting The $\delta$-Phase of Poly(vinylidene fluoride) for SolutionProcessed Ferroelectric Thin Films. Nat. Mater. 2013, 12, 433-439.

18. Hong, C. C.; Huang, S. Y.; Shieh, J.; Chen, S. H. Enhanced Piezoelectricity of Nanoimprinted Sub-20 nm Poly(vinylidene fluoride-trifluoroethylene) Copolymer Nanograss. Macromolecules 2012, 45, 1580-1586.

19. Cauda, V.; Torre, V.; Falqui, A.; Canavese, G.; Stassi, S.; Bein, T.; Pizzi, M. Confinement in Oriented Mesopores Induces Piezoelectric Behavior of Polymeric Nanowires. Chem. Mater. 2012, 24, 4215-4221.

20. Gutierrez, M. C. G.; Linares, A.; Hernandez, J. J.; Rueda, D. R.; Ezquerra, T. A.; Poza, P.; Davies, R. J. Confinement-Induced One-Dimensional Ferroelectric Polymer Arrays. Nano Lett. 2010, 10, 1472-1476. 
21. Lopes, A. C.; Costa, C. M.; Tavares, C. J.; Neves, I. C.; Mendez, S. L. Nucleation of the Electroactive $\gamma$ Phase and Enhancement of the Optical Transparency in Low Filler Content Poly(vinylidene)/Clay Nanocomposites. J. Phys. Chem. C 2011, 115, 18076-18082.

22. Yu, L.; Cebe, P. Effect of Nanoclay on Relaxation of Poly(vinylidene fluoride) Nanocomposites. J. Polym. Sci. 2009, 47, 2520-2532.

23. Kang, S. J.; Park, Y. J.; Hwang, J.; Jeong, H. J.; Lee, J. S.; Kim, K. J.; Kim, H. C.; Huh, J.; Park, C. Localized Pressure-Induced Ferroelectric Pattern Arrays of Semicrystalline Poly(vinylidene fluoride) by Microimprinting. Adv. Mater. 2007, 19, 581-586.

24. Li, M.; Stingelin, N.; Michels, J. J.; Spijkman, M. J.; Asadi, K.; Feldman, K.; Blom, P. W. M.; Leeuw, D. M. Ferroelectric Phase Diagram of PVDF:PMMA. Macromolecules 2012, 45, 7477-7485.

25. Naber, R. C. G.; Blom, P. W. M.; Marsman, A. W.; Leeuw, D. M. Low Voltage Switching of a Spin Cast Ferroelectric Polymer. Appl. Phys. Lett. 2004, 85, 2032-2034.

26. Wegener, M. Polarization-Electric Field Hysteresis of Ferroelectric PVDF Films: Comparison of Different Measurement Regimes. Rev. Sci. Instrum. 2008, 79, 106103-106106.

27. Hu, Z.; Tian, M.; Nysten, B.; Jonas, A. M. Regular Arrays of Highly Ordered Ferroelectric Polymer Nanostructures for Non-Volatile Low-Voltage Memories. Nat. Mater. 2009, 8, 62-67.

28. Koseki, Y.; Aimi, K.; Ando, S. Crystalline Structure and Molecular Mobility of PVDF Chains in PVDF/PMMA Blend Films Analyzed by Solid-State ${ }^{19}$ F MAS NMR Spectroscopy. Polym. J. 2012, 44, 757-763.

29. Oh, S.; Kim, Y.; Choi, Y. Y.; Kim, D.; Choi, H.; No, K. Fabrication of Vertically Well-Aligned P(VDF-TrFE) Nanorod Arrays. Adv. Mater. 2012, 24, 5708-5712.

30. Cha, S. N.; Kim, S. M.; Kim, H. J.; Kim, J. Y.; Ku, J. Y.; Shon, J. I.; Park, Y. J.; Song, B. G.; Jung, M. H.; Lee, E. K.; et al. Porous PVDF As Effective Sonic Wave Driven Nanogenerators. Nano Lett. 2011, 11, 5142-5147.

31. Hu, Z.; Baralia, G.; Bayot, V.; Gohy, J. F.; Jonas, A. M. Nanoscale Control of Polymer Crystallization by Nanoimprint Lithography. Nano Lett. 2005, 5, 1738-1743.

32. Leivo, E.; Wilenius, T.; Kinos, T.; Vuoristo, P.; Mantyla, T. Properties of Thermally Sprayed Fluoropolymer PVDF, ECTFE, PFA and FEP Coatings. Prog. Org. Coat. 2004, 49, 69-73.

33. Bodhane, S. P.; Shirodkar, V. S. Change in Crystallinity of Poly(vinylidene fluoride) Due to Thermal Evaporation. J. Appl. Polym. Sci. 1997, 64, 225-230.

34. Chen, S.; Li, X.; Yao, K.; Tay, F. E. H.; Kumar, A.; Zeng, K. Selfpolarized Ferroelectric PVDF Homopolymer Ultra-Thin Films Derived From Langmuire-Blodgett Deposition. Polymer 2012, 53, 1404-1408.

35. Yang, P.; Yan, R.; Fardy, M. Semiconductor Nanowire: What's Next? Nano Lett. 2010, 10, 1529-1536.

36. Wallentin, J.; Anttu, N.; Asoli, D.; Huffman, M.; Aberg, I.; Magnusson, M. H.; Siefer, G.; Kailuweit, P. F.; Dimroth, F.; Witzigmann, B.; et al. InP Nanowire Array Solar Cells Achieving $13.8 \%$ Efficiency by Exceeding the Ray Optics Limit. Science 2013, 339, 1057-1060.

37. Xie, P.; Xiong, Q.; Fang, Y.; Qing, Q.; Lieber, C. M. Local Electrical Potential Detection of DNA by NanowireNanopore Sensors. Nat. Nanotechnol. 2012, 7, 119-125.

38. Xiong, F.; Bae, M. H.; Dai, Y.; Liao, A. D.; Behnam, A.; Carrion, E. A.; Hong, S.; lelmini, D.; Pop, E. Self-Aligned NanotubeNanowire Phase Change Memory. Nano Lett. 2012, 13, 464-469.

39. Martin, J.; Mijangos, C. Tailored Polymer-Based Nanofibers and Nanotubes by Means of Different Infiltration Methods into Alumina Nanopores. Langmuir 2009, 25, 1181-1187.

40. Choi, S. W.; Kim, J. R.; Ahn, Y. R.; Jo, S. M.; Cairns, E. J. Characterization of Electrospun PVdF Fiber-Based Polymer Electrolytes. Chem. Mater. 2007, 19, 104-115.

41. Yang, Y.; Centrone, A.; Chen, L.; Simeon, F.; Hatton, T. A.; Rutledge, G. C. Highly Porous Electrospun Polyvinylidene Fluoride (PVDF)-Based Carbon Fiber. Carbon 2011, 49, 3395-3403.
42. Bayindir, M.; Sorin, F.; Abouraddy, A. F.; Viens, J.; Hart, S. D.; Joannopoulos, J. D.; Fink, Y. Metal-Insulator-Semiconductor Optoelectronic Fibres. Nature 2004, 431, 826-829.

43. Yaman, M.; Khudiyev, T.; Ozgur, E.; Kanik, M.; Aktas, O.; Ozgur, E. O.; Deniz, H.; Korkut, E.; Bayindir, M. Arrays of Indefinitely Long Uniform Nanowires and Nanotubes. Nat. Mater. 2011, 10, 494-501.

44. Khudiyev, T.; Ozgur, E.; Yaman, M.; Bayindir, M. SizeDependent Structural Coloring in Large Scale Core-Shell Nanowires. Nano Lett. 2011, 11, 4661-4665.

45. Ozgur, E.; Aktas, O.; Kanik, M.; Yaman, M.; Bayindir, M. Macroscopic Assembly of Indeinitely Long and Parallel Nanowires into Large Area Photodetection Circuitry. Nano Lett. 2012, 12, 2483-2487.

46. Egusa, S.; Wang, Z.; Chocat, N.; Ruff, Z. M.; Stolyarov, A. M.; Shemuly, D.; Sorin, F.; Rakich, P. T.; Joannopoulos, J. D.; Fink, Y. Multimaterial Piezoelectric Fibres. Nat. Mater. 2010, 9, 643-648.

47. Horiuchi, S.; Hanada, T.; Ebisawa, M.; Matsuda, Y.; Kobayashi, M.; Takahara, A. Contamination-Free Transmission Electron Microscopy for High-Resolution Carbon Elemental Mapping of Polymers. ACS Nano 2009, 3, 1297-1304.

48. Suman, B.; Tandon, P. Fluid Flow Stability Analysis of Multilayer Fiber Drawing. Chem. Eng. Sci. 2010, 65, 55375549.

49. Pu, J.; Yan, X.; Jiang, Y.; Chang, C.; Lin, L. Piezoelectric Actuation of Direct-Write Electrospun Fibers. Sens. Actuators, $A$ 2010, 164, 131-136.

50. Zhao, M. H.; Wang, Z. L.; Mao, S. X. Piezoelectric Characterization of Individual Zinc Oxide Nanobelt Probed by Piezoresponse Force Microscope. Nano Lett. 2004, 4, 587-590.

51. Hohenberg, P.; Kohn, W. Inhomogeneous Electron Gas. Phys. Rev. 1964, 136, B864.

52. Kohn, W.; Sham, L. J. Self-Consistent Equations Including Exchange and Correlation Effects. Phys. Rev. 1965, 140, A1133.

53. Kresse, G.; Hafner, J. Ab Initio Molecular Dynamics for Liquid Metals. Phys. Rev. B: Condens. Matter Mater. Phys. 1993, 47, 558.

54. Kresse, G.; Furthmüller, J. Efficient Iterative Schemes for $A b$ Initio Total-Energy Calculations Using a Plane-Wave Basis Set. Phys. Rev. B: Condens. Matter Mater. Phys. 1996, 54, 11169.

55. Perdew, J. P.; Burke, K.; Ernzerhof, M. Generalized Gradient Approximation Made Simple. Phys. Rev. Lett. 1996, 77, 3865.

56. Blöchl, P. E. Projector Augmented-Wave Method. Phys. Rev. B: Condens. Matter Mater. Phys. 1994, 50, 17953. 